\title{
Solar Radius at Sub-Terahertz Frequencies
}

\author{
Fabian Menezes and Adriana Valio \\ Center for Radio Astronomy and Astrophysics (CRAAM) \\ Mackenzie Presbyterian University, São Paulo, Brazil \\ email: fabianme17@gmail.com \\ email: avalio@craam.mackenzie.br
}

\begin{abstract}
The visible surface of the Sun, or photosphere, is defined as the solar radius in the optical spectrum range located at $696,000 \mathrm{~km}$ (Cox et al. (Ed. 2015)). However, as the altitude increases, the dominant electromagnetic radiation is emitted at other frequencies. Our aim is to measure the solar radius at frequencies of $212 \mathrm{GHz}$ and $405 \mathrm{GHz}$ through out a solar cycle and, therefore, the altitude where these emissions are generated and that variation along the years. Also we tried to verify the the radius dependence on the solar activity cycle, which can be a good indicator of the changes that occur in the atmosphere structure. For this, we used data obtained by the Submillimetric Solar Telescope (SST) created from daily scans made by SST from 1990 to 2015. From these scans a 2D map of the solar disk was constructed. The solar radius is then determined by adjusting a circumference to the points where the brightness is half of the quiet Sun level, which is set as the most common temperature value in the solar map, i.e., the mode of the temperature distribution. Thus, we determined the solar radius at 212 and $405 \mathrm{GHz}$ and the altitude of the emissions respectively. For $212 \mathrm{GHz}$, we obtained a radius of $976.5 " \pm 8 "(707 \pm 4 \mathrm{Mm})$, whereas for $405 \mathrm{GHz}$, we obtained $975.0 " \pm 8 "$ (707 $\pm 5 \mathrm{Mm})$. optical spectrum range
\end{abstract}

Keywords. Sun: activity, Sun: chromosphere, Sun: fundamental parameters, Sun: radio radiation

\section{Introduction}

The Sun is considered a mildly active star with an 11-year activity cycle. It emits radiation across the electromagnetic spectrum at several wavelengths, from gamma rays to radio waves.

Our aim is to measure the solar radius at frequencies of $212 \mathrm{GHz}$ and $405 \mathrm{GHz}$ and, therefore, the altitude where these emissions are generated. The relevance of this research is the possibility to understand more about the solar atmosphere and what is the radius dependence on the solar activity cycle, which can be a good indicator of the changes that occur in the atmosphere structure.

\section{Sub-terahertz observations}

We used data obtained by the Submillimetric Solar Telescope (SST), in the Astronomical Complex El Leoncito Observatory (CASLEO) at the Argentinean Andes in partnership with the Center for Radio-Astronomy and Astrophysics Mackenzie (CRAAM). This telescope uses a multibeam system operating at radio frequencies with 4 beams at 212 $\mathrm{GHz}$ and 2 beams at $405 \mathrm{GHz}$ (Figure 1a). Maps of the whole Sun were created (Figure 1b) from daily scans made by SST from 1999 to 2015 (Figure 2).

The the quiet Sun level is set as the most common temperature value in the solar map, i.e., the mode of the temperature distribution (Figure 3a). Next, the solar limb is set as the half of the quiet Sun level (Figure $3 \mathrm{~b}$ ). Then, the solar radius is obtained by 

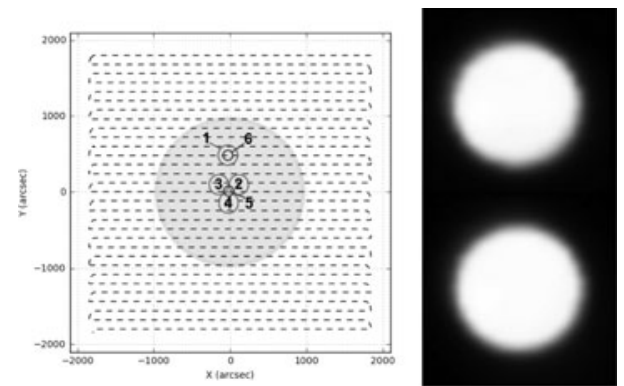

Figure 1. Left: scans over the solar disk made by SST. The colored circles represent the telescope beams. Right: examples of solar maps made from SST's data.
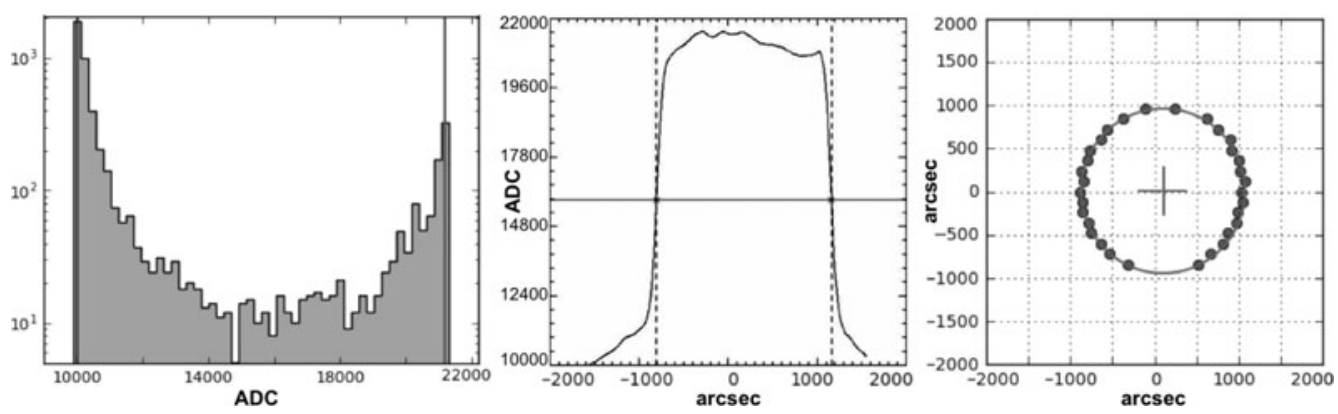

Figure 3. Left: histogram of intensities where we define the value of quiet Sun. Middle: the markup of the limb points. Right: the coordinates of the limb with the circumference fit.

Table 1. Solar radius results for $212 \mathrm{GHz}$ and $405 \mathrm{GHz}$ frequencies

\begin{tabular}{lccc}
\hline Frequency & Radius $(\operatorname{arcsec})$ & Radius $(\mathrm{Mm})$ & Altitude $(\mathrm{km})$ \\
\hline $212 \mathrm{GHz}$ & $974 \pm 5$ & $707 \pm 4$ & 11,492 \\
$405 \mathrm{GHz}$ & $974 \pm 7$ & $707 \pm 5$ & 11,492 \\
Optical & 959.63 & $695.508 \pm 0.026$ & 0 \\
\hline
\end{tabular}

adjusting a circumference to the solar limb points (Figure 3c). Thus, by substracting the $\mathrm{sub}-\mathrm{THz}$ radius from the visible one, we are able to determine the altitudes where the $212 \mathrm{Ghz}$ and $405 \mathrm{GHz}$ emissions are generated.

\section{Preliminary results}

For $212 \mathrm{GHz}$, we obtained a radius of $974 " \pm 5 "(707 \pm 4 \mathrm{Mm})$, whereas for $405 \mathrm{GHz}$, we obtained a radius of $974 " \pm 7 "(707 \pm 5 \mathrm{Mm})$. These results are shown in Table 1 and Figure 4 .

In addition to the mean solar radius results at both frequencies, we investigated the relationship of the solar radius variation at submillimetric wavelengths with the solar activity cycle of 11 years. Unfortunately it was not possible to observe this relationship due to high dispersion of data. 

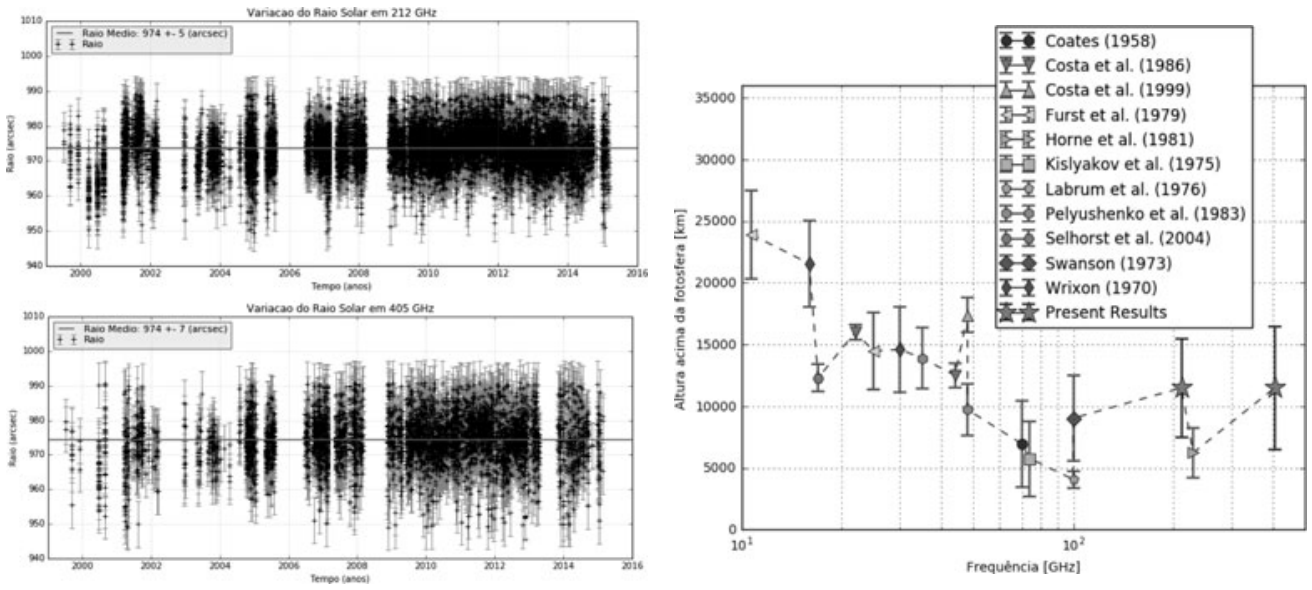

Figure 4. Fitted radius of the solar disk throughout the solar cycle at 212 $\mathrm{GHz}$ (above) and $405 \mathrm{GHz}$ (below).

Figure 5. Height of emission above the photosphere as function of the frequency with previous results from other authors.

\section{Conclusion}

From the radii obtained - from the fit to the SST maps (Figure 4) - we could calculate their respective heights above the photosphere (Table. 1): the 212-GHz emission is produced at an altitude of $11,492 \pm 4000 \mathrm{~km}$, whereas $405-\mathrm{GHz}$ one is emitted at an altitude of $11,492 \pm 5000 \mathrm{~km}$. In Figure 5 we can also compare our present rusults with previous ones from Coates (1958), Costa et al. (1986), Costa et al. (1999), Fürst et al. (1979), Horne et al. (1981), Kisliakov et al. (1975), Labrum et al. (1978), Pelyushenko \& Chernyshev (1983), Selhorst et al. (2004), Swanson, P. N. (1973), Wrixon (1970).

The Sun's atmosphere is divided to three structures. According to Cox et al. (Ed. 2015), they are photosphere (from 0 to $525 \mathrm{~km}$ ), chromosphere (from 525 to $2100 \mathrm{~km}$ ) and the corona (from $2100 \mathrm{~km}$ and above). So, we can conclude that both emission frequencies are dominant at the corona layer.

\section{Aknowledgements}

Special thanks to MackPesquisa and IAU for the support and aid for the this event (IAUS 328) and to CAPES for the scholarship aimed at the postgraduate program of Geospatial Sciences and Applications.

\section{References}

Coates, R. J. 1958, The Astrophysical Journal, 128, 83.

Costa, J. E. R., Homor, J. L., \& Kaufmann, P. 1986, In Solar Flares $\&$ Coronal Physics Using P/OF as a Research Tool, 1, 201.

Costa, J. E. R., \& Silva, A. V. R., \& Makhmutov, V. S., \& Rolli, E., \& Kaufmann, P., \& Magun, A. 1999, The Astrophysical Journal Letters, 520, L63.

Cox, A. N. and other authors (Ed.) 2015, Allen's astrophysical quantities.

Fürst, E., Hirth, W., \& Lantos, P. 1979, Solar Physics, 63(2), 257.

Horne, K., Hurford, G. J., Zirin, H., \& De Graauw, T. 1981, The Astrophysical JournaL, 244, 340.

Kisliakov, A. G., Kulikov, I. I., Fedoseev, L. I., \& Chernyshev, V. I. 1975, Soviet Astronomy Letters, 1, 79.

Labrum, N. R., Archer, J. W., \& Smith, C. J. 1978, Solar Physics, 59(2), 331. 
Pelyushenko, S. A. \& Chernyshev, V. I. 1983, Soviet Astronomy, 27, 340.

Selhorst, C. L., \& Silva, A. V. R., \& Costa, J. E. R. 2004, Astronomy \&3 Astrophysics, 420, 1117.

Swanson, P. N. 1973, Solar Physics, 32(1), 77.

Wrixon, G. T. 1970, Nature, 227, 1231. 